\title{
Ultra-Low-Voltage CMOS-Based Current Bleeding Mixer with High LO-RF Isolation
}

\author{
Gim Heng Tan, ${ }^{1,2}$ Roslina Mohd Sidek, ${ }^{1}$ Harikrishnan Ramiah, \\ Wei Keat Chong, ${ }^{3}$ and De Xing Lioe ${ }^{1}$ \\ ${ }^{1}$ Department of Electrical and Electronic Engineering, Universiti Putra Malaysia, 43400 Serdang, Malaysia \\ ${ }^{2}$ Department of Electrical and Electronic Engineering, Segi University, 47810 Petaling Jaya, Selangor, Malaysia \\ ${ }^{3}$ Department of Electrical Engineering, University of Malaya, 50603 Kuala Lumpur, Malaysia
}

Correspondence should be addressed to Harikrishnan Ramiah; hrkhari@um.edu.my

Received 13 June 2014; Accepted 27 July 2014; Published 14 August 2014

Academic Editor: Changzhi Li

Copyright (C) 2014 Gim Heng Tan et al. This is an open access article distributed under the Creative Commons Attribution License, which permits unrestricted use, distribution, and reproduction in any medium, provided the original work is properly cited.

\begin{abstract}
This journal presents an ultra-low-voltage current bleeding mixer with high LO-RF port-to-port isolation, implemented on $0.13 \mu \mathrm{m}$ standard CMOS technology for ZigBee application. The architecture compliments a modified current bleeding topology, consisting of NMOS-based current bleeding transistor, PMOS-based switching stage, and integrated inductors achieving lowvoltage operation and high LO-RF isolation. The mixer exhibits a conversion gain of $7.5 \mathrm{~dB}$ at the radio frequency $(\mathrm{RF}) \mathrm{of} 2.4 \mathrm{GHz}$, an input third-order intercept point (IIP3) of $1 \mathrm{dBm}$, and a LO-RF isolation measured to $60 \mathrm{~dB}$. The DC power consumption is $572 \mu \mathrm{W}$ at supply voltage of $0.45 \mathrm{~V}$, while consuming a chip area of $0.97 \times 0.88 \mathrm{~mm}^{2}$.
\end{abstract}

\section{Introduction}

Various low-power front-end receivers had been widely reported for application such as wireless sensor network (WSN). WSN application which requires low-power operation often adopts ZigBee standard with the operating frequency ranging from 2.4 to $2.4835 \mathrm{GHz}$ [1]. Direct conversion receiver (DCR) has been in the heat of discussion in recent years due to its inherent low power consumption and the simplicity in realization [2]. One of the major setbacks associated with DCR is the LO to RF port-to-port isolation. An incurred mismatch in the device physical dimension would potentially couple the LO leakage to the RF port through the gate-drain capacitance, $C_{\mathrm{gd}}$ of the RF transconductance transistors. The leakage component will mix with RF signal, resulting in a detrimental phenomenon known as self-mixing [3] which in effect produces DC offset that degrades the performance of the overall receiver. In preference, high isolation between the LO-RF ports is crucial in alleviating self-mixing. The typical LO-RF isolations of the standard Gilbert cell mixer are in the range of $40-50 \mathrm{~dB}$ [4]. In this paper, the conventional current bleeding architecture which has a high conversion gain and low noise figure is modified by integrating a combination of NMOS-based current bleeding transistor, PMOS-based LO switch, and integrated inductors, thus improving the isolation between the $\mathrm{LO}$ and RF port.

\section{Proposed Design}

Previous design: Figure 1 shows the conventional CMOS current bleeding mixer that integrates a combination of PMOS-based current bleeding stage and NMOS-based local oscillator, LO switching stage [5].

In effect to the mismatch in the switching stage, the LO leakage component at nodes $X_{1}$ and $X_{2}$ will directly couple to the RF port through the gate-drain capacitance, $C_{\mathrm{gd}}$, of the RF transconductance stage $\left(M_{1}-M_{2}\right)$. This will adversely reduce the isolation between the LO and RF ports.

Figure 2 shows that the proposed mixer consists of a RF transconductance stage $\left(M_{1}-M_{2}\right)$, a PMOS-based LO switching input $\left(M_{3}-M_{6}\right)$, a NMOS-based current bleeding stage $\left(M_{7}-M_{8}\right)$, and the output load $\left(R_{L 1}-C_{L 1}\right.$ and $\left.R_{L 2}-C_{L 2}\right)$. Inductors $L_{d 1}$ and $L_{d 2}$ act as a RF choke in alleviating the RF 


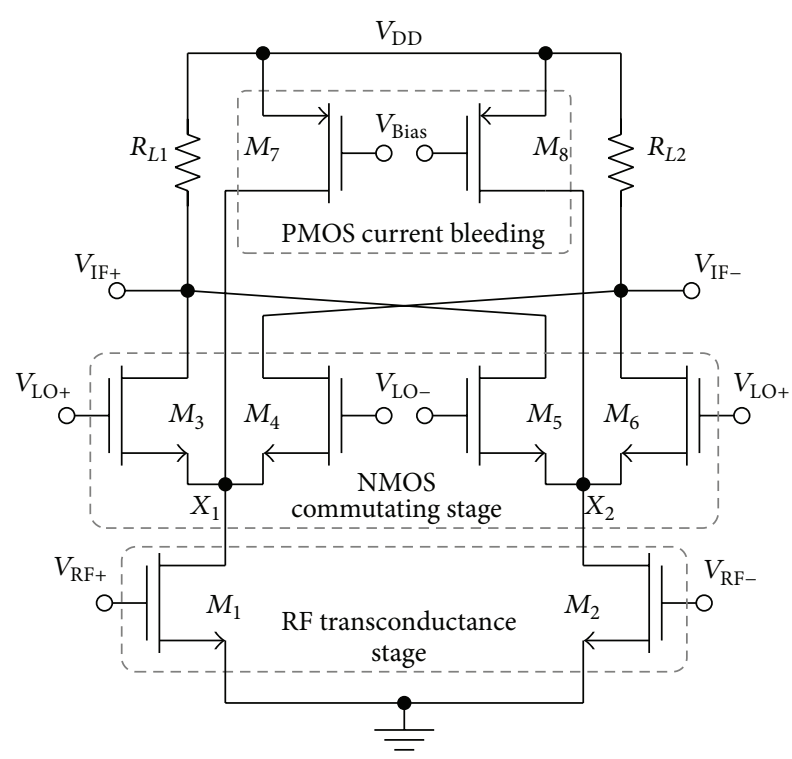

FIgURE 1: Conventional current bleeding mixer.

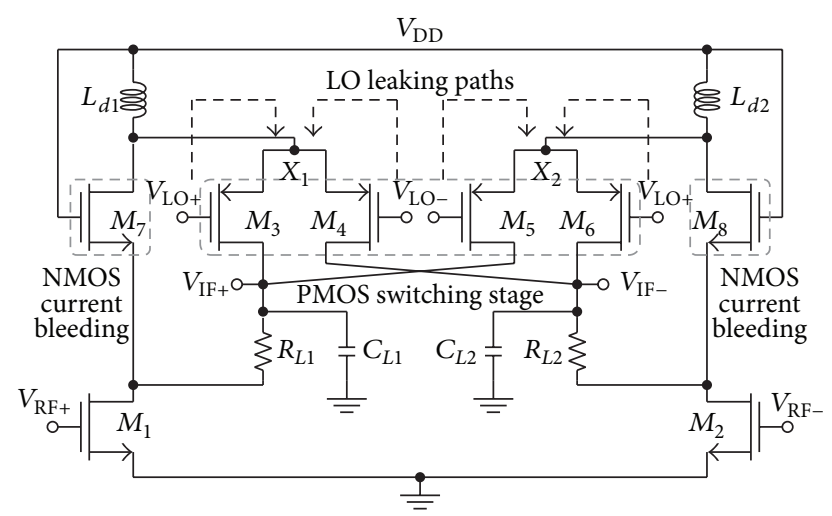

Figure 2: A schematic view of the proposed mixer.

signal leakage into the voltage supply, $V_{\mathrm{DD}}$. The RF frequency is mixed with the LO frequency at node $X_{1}$ and $X_{2}$. The differential output current, neglecting the higher order spurs, can be derived as follows:

$$
\begin{aligned}
i_{\mathrm{IF}}= & \frac{2}{\pi} \cdot g_{m 1,2} \\
& \cdot v_{\mathrm{RF}}\left[\sin \left(\omega_{\mathrm{RF}}-\omega_{\mathrm{LO}}\right) t-\sin \left(\omega_{\mathrm{RF}}+\omega_{\mathrm{LO}}\right) t\right],
\end{aligned}
$$

where $g_{m 1,2}$ is the transconductance for $M_{1}$ and $M_{2}$ and $v_{\mathrm{RF}}$ is the input RF signal, while $\omega_{\mathrm{RF}}$ and $\omega_{\mathrm{LO}}$ are the RF and LO frequency, respectively. At the IF output, the combination of $R_{L 1}-C_{L 1}$ and $R_{L 2}-C_{L 2}$ forms a low pass filter (LPF), which filters out the high-order spurs at the output such as the upconverted frequency component $\sin \left(\omega_{\mathrm{RF}}+\omega_{\mathrm{LO}}\right)$.

An incurred mismatch in the LO switching transistor physical dimension would result in a feed-through of LO leakage at nodes $X_{1}$ and $X_{2}$ to the RF port as described in Figure 2. The dotted arrowhead illustrates the LO leakage path from the LO ports to nodes $X_{1}$ and $X_{2}$.
Other than for providing a desirable bleeding path for the DC current, transistor $M_{7}-M_{8}$ is optimized to enhance the LO-RF isolation. Transistors $M_{7}$ and $M_{1}$ are cascoded in series, thus observing high impedance, $R_{X}$ referring to the drain terminal of the bleeding transistor $M_{7}$, expressed as

$$
R_{x} \approx g_{m 7} \cdot r_{o 1} \cdot r_{o 7}
$$

where $g_{m 7}$ and $r_{o 7}$ are the transconductance and output resistance for $M_{7}$ while $r_{o 1}$ is the output resistance for transistor $M_{1}$. Accordingly, this high impedance node minimizes the LO leakage from nodes $X_{1}$ and $X_{2}$ to the RF port. As a bench of comparison to the design in Figure 1, the LO leakage component at nodes $X_{1}$ and $X_{2}$ would directly couple to the RF port through parasitic capacitance $C_{\mathrm{gd}}$ in the absence of additional shielding between LO-RF port. The proposed mixer on the other hand utilizes the bleeding transistor $\left(M_{7}\right.$ and $\left.M_{8}\right)$ as the shielding element between LO and RF port to improve the LO-RF isolation. In addition, the high frequency LO leakage at the output node of $V_{\mathrm{IF}+}$ and $V_{\mathrm{IF}-}$ as shown in Figure 2 is insignificant as it is directed towards the ground rail via the low impedance path of capacitor $C_{L 1}$ and $C_{L 2}$ in contrary to the conventional architecture where the leakage component couples to the RF port. Additionally any LO leakage at the IF output port is further attenuated by load resistor $R_{L(1,2)}$ before reaching the RF port, in a goal of improving the LO-RF isolation.

Along with the presence of the cascoded configuration between transconductance and the bleeding stage, this mixer is able to work down to $0.45 \mathrm{~V}$ of supply headroom. The conventional current bleeding mixer as shown in Figure 1 requires an $\mathrm{LO}$ bias of

$$
V_{\mathrm{LO}}=V_{\mathrm{gs} 3}+V_{\mathrm{ds} 1(\mathrm{sat})}
$$

where $V_{\mathrm{LO}}$ is the DC voltage to bias the switching transistors, $V_{\mathrm{gs} 3}$ is the gate to source voltage of transistor $M_{3}$, and $V_{\mathrm{ds} 1 \text { (sat) }}$ is the overdrive voltage of transistor $M_{1}$. By adapting PMOSbased LO switching stage $\left(M_{3}-M_{6}\right)$, coupled together with inductors $L_{d 1}$ and $L_{d 2}$ as illustrated in Figure 2, the DC voltage required to bias the gate of transistor $M_{3}-M_{6}$ is reduced to only $V_{\text {sg }}$ (source-gate voltage) which approximate to the threshold voltage, $V_{\text {th }}$ of the PMOS transistor, whereas the DC voltage at nodes $X_{1}$ and $X_{2}$ approaches to $V_{\mathrm{DD}}$. The LO bias voltage $V_{\mathrm{LO}}$ is given as

$$
V_{\mathrm{LO}}=V_{\mathrm{dd}}-V_{\mathrm{th} 3},
$$

where $V_{\text {th3 }}$ is the threshold voltage of transistor $M_{3}$. The DC voltage required to turn on the LO switching stage no longer depended on the overdrive voltage, $V_{\mathrm{ds1} 12 \text { (sat) }}$ of the transconductance stage $\left(M_{1}-M_{2}\right)$ as given in (3). In this proposed mixer, the DC voltage for $V_{\mathrm{LO}}$ nears ground potential instead of the positive power rail resulting the design to operate favorably at ultra-low supply headroom. As for the conventional current bleeding mixer architecture as in Figure 1, the DC bias voltage for $V_{\mathrm{LO}}$ moves towards the positive rail providing a bottleneck in operating the mixer at low supply headroom. 


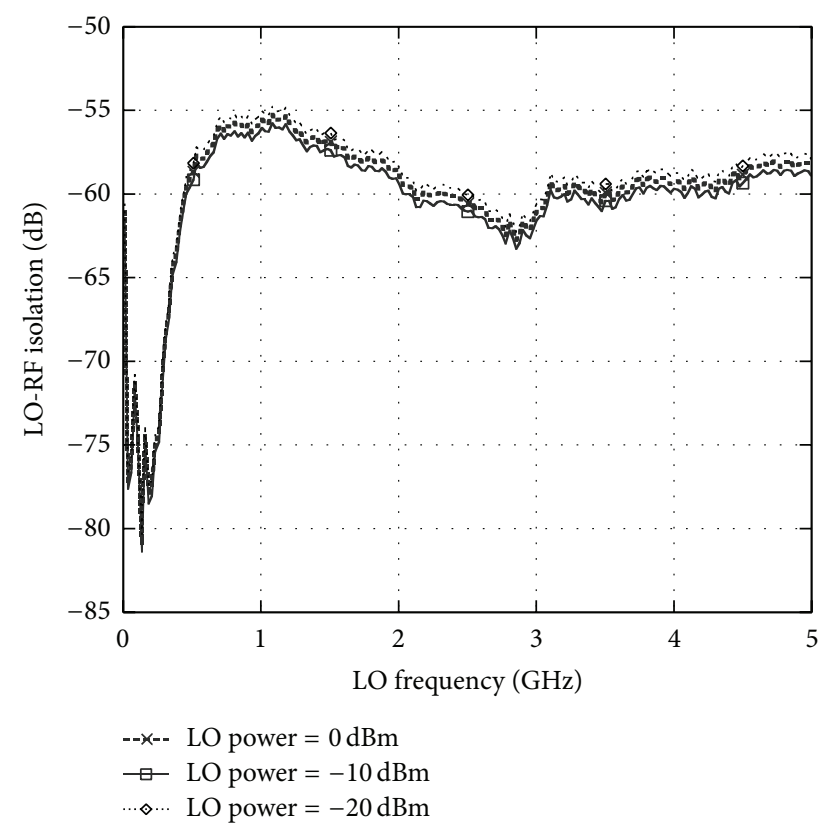

FIgURE 3: Measured LO-RF isolation.

\section{Measurement Result}

The proposed mixer is implemented on a $0.13 \mu \mathrm{m}$ standard CMOS technology. The mixer consumes only $1.27 \mathrm{~mA}$ of DC current from $0.45 \mathrm{~V}$ of supply voltage. The LO-RF isolation in $\mathrm{dB}$ is given as [10]

$$
P_{\text {Isolation }}(\mathrm{dBm})=P_{\text {flo }(\mathrm{LO})}(\mathrm{dBm})-P_{\mathrm{flo}(\mathrm{RF})}(\mathrm{dbm}),
$$

where $P_{\text {Isolation }}$ is the isolation between LO and RF port due to the leakage component from LO port, $P_{\text {flo(LO) }}$ is the injected LO power at LO port, and $P_{\text {flo(RF) }}$ is the observed LO power coupled to the RF port. The LO-RF isolation has been measured at a difference discrete LO power as described in Figure 3. It can be observed that from the frequency of $2 \mathrm{GHz}$ to $5 \mathrm{GHz}$, the isolation achieved is more than $55 \mathrm{~dB}$ and at $2.4 \mathrm{GHz}$, the LO-RF isolation is measured at $60 \mathrm{~dB}$. The isolation technique adapted in this circuit has improved the LO-RF shielding significantly while operating at ultra-low supply voltage down to $0.45 \mathrm{~V}$. Figure 4 shows the results for LO-IF isolation which measures more than $64 \mathrm{~dB}$ at $2.4 \mathrm{GHz}$. Two-tone test with an input frequency of $2.443 \mathrm{GHz}$ and $2.442 \mathrm{GHz}$ is applied to the RF port with the corresponding LO frequency of $2.439 \mathrm{GHz}$ to quantify the linearity of the mixer. The conversion gain of the proposed mixer is observed to be $7.5 \mathrm{~dB}$ with an IIP3 of $1 \mathrm{dBm}$ as shown in Figure 5. Inductors $L_{d 1}$ and $L_{d 2}$ in the proposed mixer of Figure 2 not only function as the DC current source but concurrently resonate out the parasitic capacitance at nodes $X_{1}$ and $X_{2}$ to improve the IIP3. The noise figure (NF) is observed to be around $18 \mathrm{~dB}$ as illustrated in Figure 6. Table 1 summarizes the design parameters for the proposed mixer and the performance comparison of the proposed architecture respective to other reported works is given in Table 2. The designed mixer has the highest LO-RF isolation

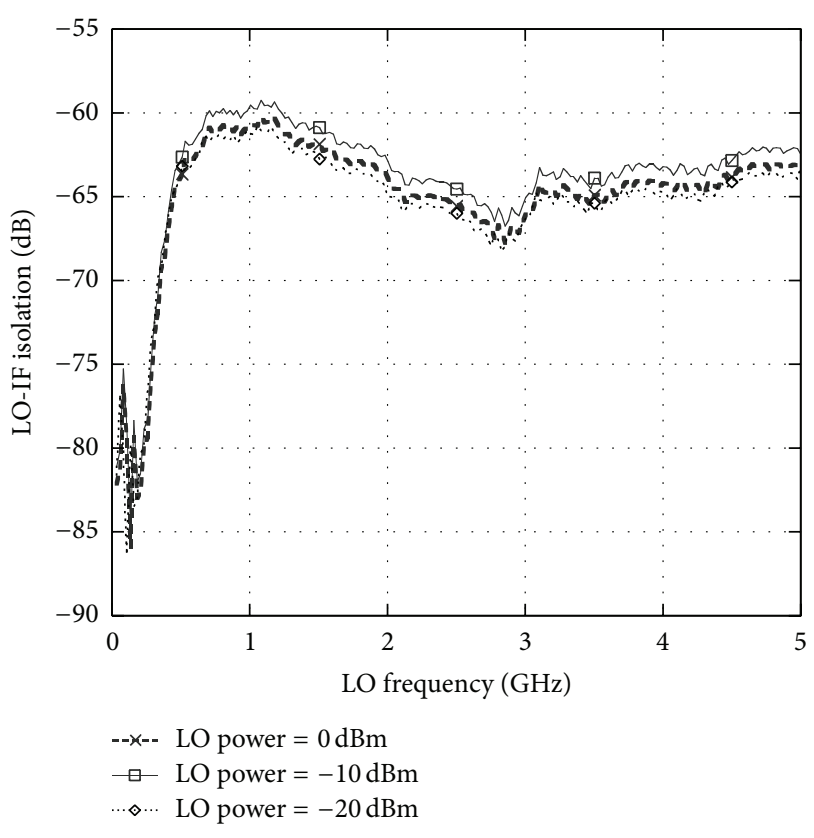

FIGURE 4: Measured LO-IF isolation.

TABLE 1: Design parameters for the mixer.

\begin{tabular}{lc}
\hline Parameters & Design values \\
\hline$M_{1}, M_{2}$ & $106 \mu \mathrm{m} / 0.13 \mu \mathrm{m}$ \\
$M_{3}, M_{4}, M_{5}, M_{6}$ & $64 \mu \mathrm{m} / 0.13 \mu \mathrm{m}$ \\
$M_{7}, M_{8}$ & $200 \mu \mathrm{m} / 0.13 \mu \mathrm{m}$ \\
$L_{d 1}, L_{d 2}$ & $6.7 \mathrm{nH}$ \\
$C_{1}, C_{2}$ & $10 \mathrm{pF}$ \\
$R_{L 1}, R_{L 2}$ & $1 \mathrm{k} \Omega$ \\
\hline
\end{tabular}

and among the lowest in DC power consumption. The measured conversion gain and linearity, IIP3, of the mixer is $7.5 \mathrm{~dB}$ and $1 \mathrm{dBm}$, respectively. The dynamic performance of the architecture is evaluated adapting a figure of merit (FOM) expression, which is highlighted in the following equation, given as [11]:

$$
\mathrm{FOM}=10 \log \left(\frac{10^{G / 20} \cdot 10^{(\mathrm{IIP} 3-10) / 20}}{10^{\mathrm{NF} / 10} \cdot P}\right)
$$

where $G$ is the conversion gain in $\mathrm{dB}$, IIP3 is the third order linearity in $\mathrm{dBm}, \mathrm{NF}$ is the noise figure in $\mathrm{dB}$, and $P$ is the power in $\mathrm{mW}$. In reasoning out the performance comparison respective to other reported recent work, the proposed architecture exhibits the highest FOM of 16.67 while relating to power dissipation well below $1 \mathrm{~mW}$. In the loop of recent reported work, the proposed architecture process to be the lowest in power consumption. The photomicrograph of the chip is illustrated in Figure 7, with a corresponding chip area of $0.97 \times 0.88 \mathrm{~mm}^{2}$. 


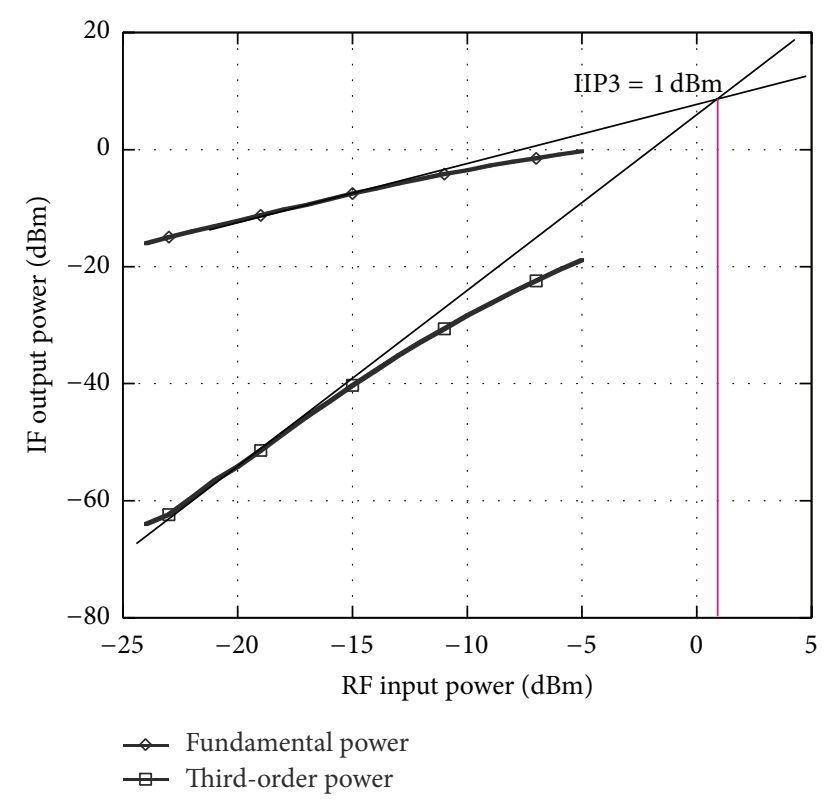

FIGURE 5: IIP3 of the proposed mixer.

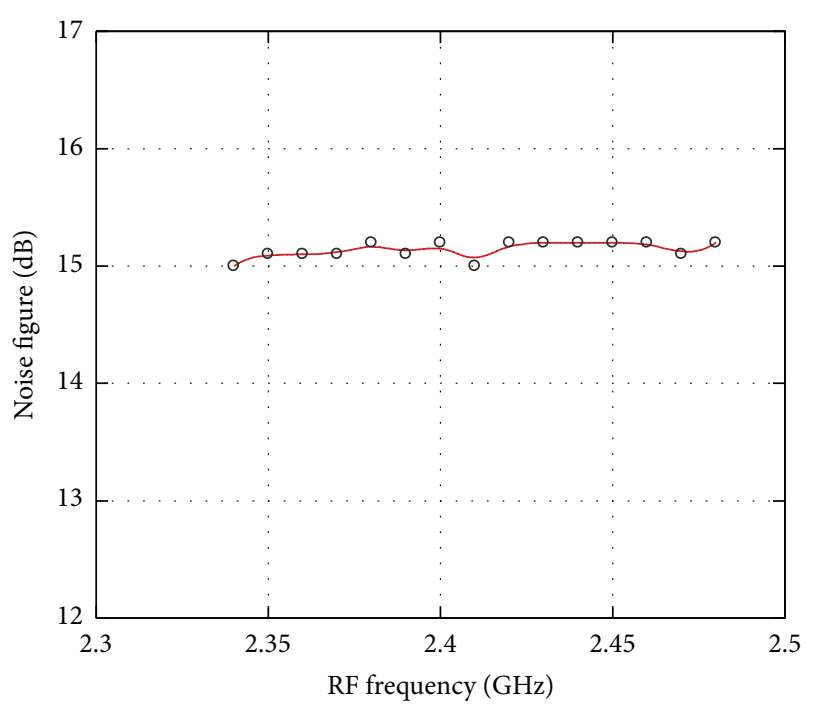

Figure 6: Noise figure.

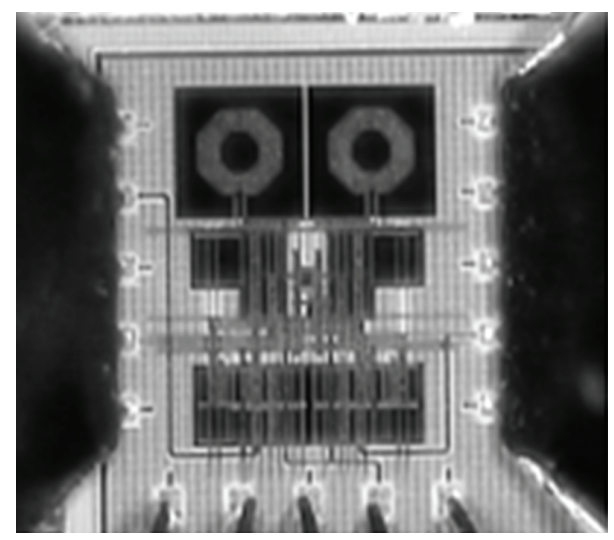

FIGURE 7: A chip micrograph of the proposed mixer.
TABLE 2: Performance summary and comparison.

\begin{tabular}{lccccc}
\hline Parameter & This work & {$[6]$} & {$[7]$} & {$[8]$} & {$[9]$} \\
\hline Supply voltage $(\mathrm{V})$ & 0.45 & 2.5 & 1.5 & 1 & 1 \\
LO frequency $(\mathrm{GHz})$ & 2.4 & 2.525 & 2.4 & 2.4 & 2.4 \\
CG $(\mathrm{dB})$ & 7.5 & 9.5 & -3.5 & 15.7 & 5.3 \\
IIP3 $(\mathrm{dBm})$ & 1 & -7.5 & 0 & -9 & 4.6 \\
LO-RF isolation $(\mathrm{dB})$ & 60 & 48 & 16.8 & 33 & - \\
NF $(\mathrm{dB})$ & 15 & - & - & 18.3 & 21.7 \\
Power $(\mathrm{mW})$ & 0.572 & 17.5 & 2 & 0.5 & 3.5 \\
FOM & 16.67 & - & - & 13.06 & 2.81 \\
Area $\left(\mathrm{mm}^{2}\right)$ & $0.9 \times 0.8$ & - & - & $1 \times 0.8$ & $0.4 \times 0.5$ \\
CMOS technology $(\mu \mathrm{m})$ & 0.13 & 0.18 & 0.35 & 0.13 & 0.18 \\
\hline
\end{tabular}

\section{Conclusion}

The proposed mixer is successfully designed and verified in $0.13 \mu \mathrm{m}$ standard CMOS technology. The implemented CMOS-based current bleeding mixer topology, which consists of a combination of NMOS-based current bleeding transistor, PMOS-based switching stage, and integrated inductors, has significantly improved the LO-RF isolation while operating at supply voltage headroom down to $0.45 \mathrm{~V}$. The design observes a considerable high LO-RF isolation of $60 \mathrm{~dB}$ and consuming merely $572 \mu \mathrm{w}$ of power, which is a promising performance metric for ZigBee application.

\section{Conflict of Interests}

The authors declare that there is no conflict of interests regarding the publication of this paper.

\section{Acknowledgment}

This research is supported by the UM High Impact Research Grant UM.C/HIR/MOHE/ENG/51 from the Ministry of Higher Education Malaysia.

\section{References}

[1] G. H. Tan, R. M. Sidek, H. Ramiah, and W. K. Chong, "Design of ultra-low voltage $0.5 \mathrm{~V}$ CMOS current bleeding mixer," IEICE Electronics Express, vol. 9, no. 11, pp. 990-997, 2012.

[2] T. Song, H.-S. Oh, E. Yoon, and S. Hong, "A low-power 2.4-GHz current-reused receiver front-end and frequency source for wireless sensor network," IEEE Journal of Solid-State Circuits, vol. 42, no. 5, pp. 1012-1022, 2007.

[3] B. Razavi, "Design considerations for direct-conversion receivers," IEEE Transactions on Circuits and Systems, vol. 44, no. 6, pp. 428-435, 1997.

[4] H. Chiou, K. Lin, and W. Chen, "A 1-V 5-GHz self-bias foldedswitch mixer in 90-nm CMOS for WLAN receiver," IEEE Transactions on Circuits and Systems, vol. 59, no. 6, pp. 12151227, 2012.

[5] J. Park, S. Member, C.-H. Lee, B.-S. Kim, and J. Laskar, “Design and analysis of low flicker-noise CMOS mixers for directconversion receivers," IEEE Transactions on Microwave Theory and Techniques, vol. 54, no. 12, pp. 4372-4380, 2006. 
[6] H.-M. Hsu and T.-H. Lee, "High LO-RF isolation of zero-IF mixer in $0.18 \mu \mathrm{m}$ CMOS technology," Analog Integrated Circuits and Signal Processing, vol. 49, no. 1, pp. 19-25, 2006.

[7] W. Liou, M. Yeh, C. Tsai, and S. Chang, "Design and implementation of a low-voltage 2.4-GHZ CMOS RF receiver frontend for wireless communication," Journal of Marine Science and Technology, vol. 13, no. 3, pp. 170-175, 2005.

[8] H. Lee and S. Mohammadi, "A $500 \mu \mathrm{W} 2.4 \mathrm{GHz}$ CMOS subthreshold mixer for ultra low power applications," in Proceedings of the IEEE Radio Frequency Integrated Circuits Symposium (RFIC '07), pp. 325-328, Honolulu, Hawaii, USA, June 2007.

[9] B. Wei and Y. Dai, "Analysis and design of a 1.0-V CMOS mixer based on variable load technique," Microelectronics Journal, vol. 43, no. 12, pp. 1003-1009, 2012.

[10] H. Ramiah, J. Kanesan, and T. Z. A. Zulkifli, "A CMOS upconversion mixer in $0.18 \mu \mathrm{m}$ technology for IEEE 802.11a WLAN application," IETE Journal of Research, vol. 59, no. 4, pp. 454-460, 2013.

[11] K.-H. Liang and H.-Y. Chang, " $0.5-6 \mathrm{GHz}$ low-voltage lowpower mixer using a modified cascode topology in $0.18 \mu \mathrm{m}$ CMOS technology," IET Microwaves, Antennas \& Propagation, vol. 5, no. 2, pp. 167-174, 2011. 

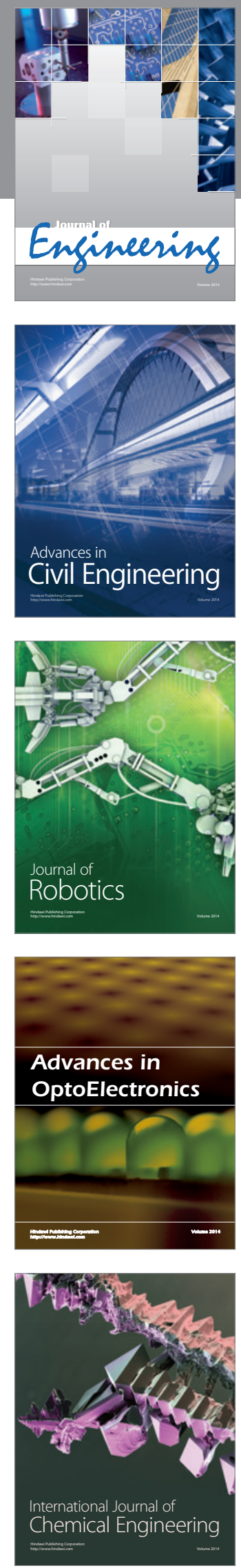

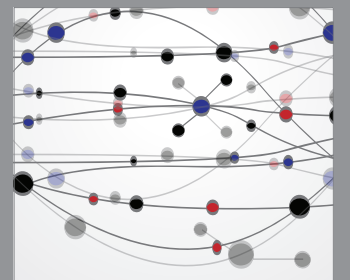

The Scientific World Journal
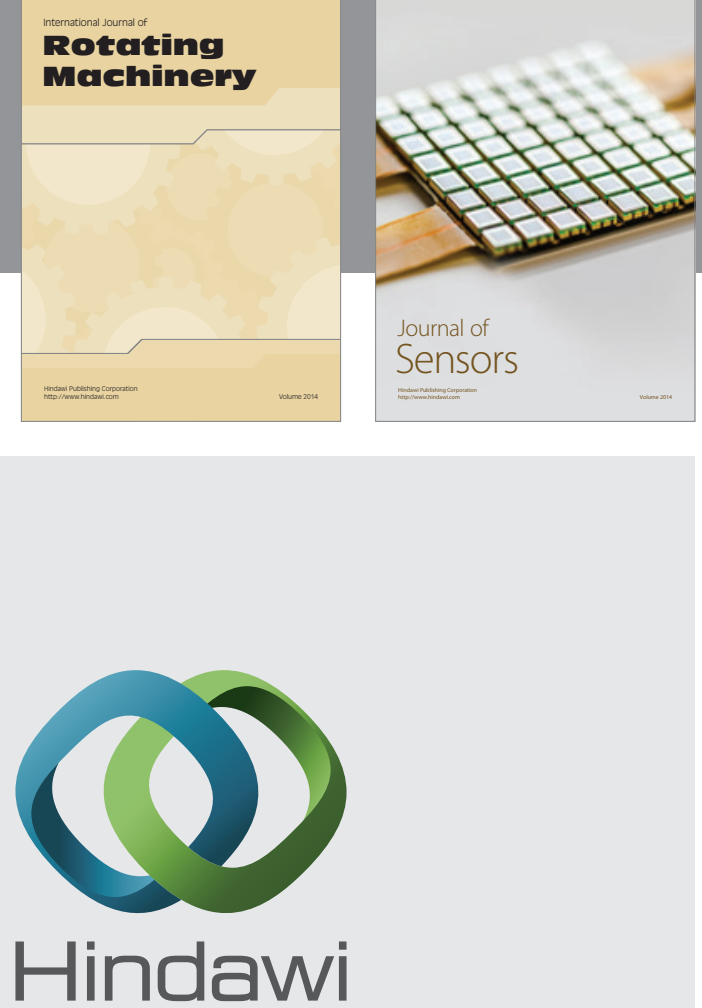

Submit your manuscripts at http://www.hindawi.com
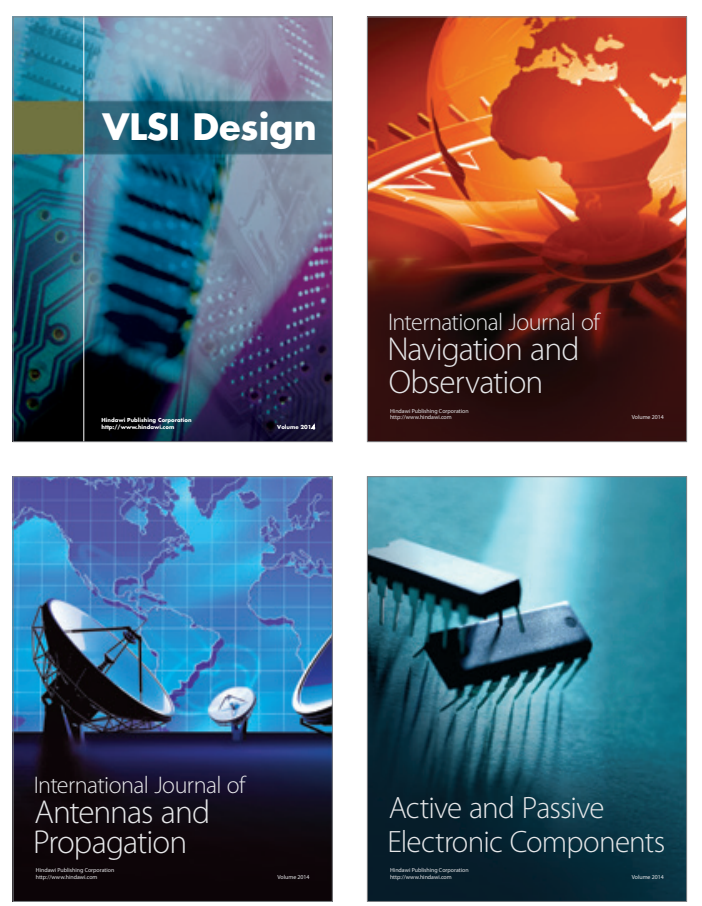
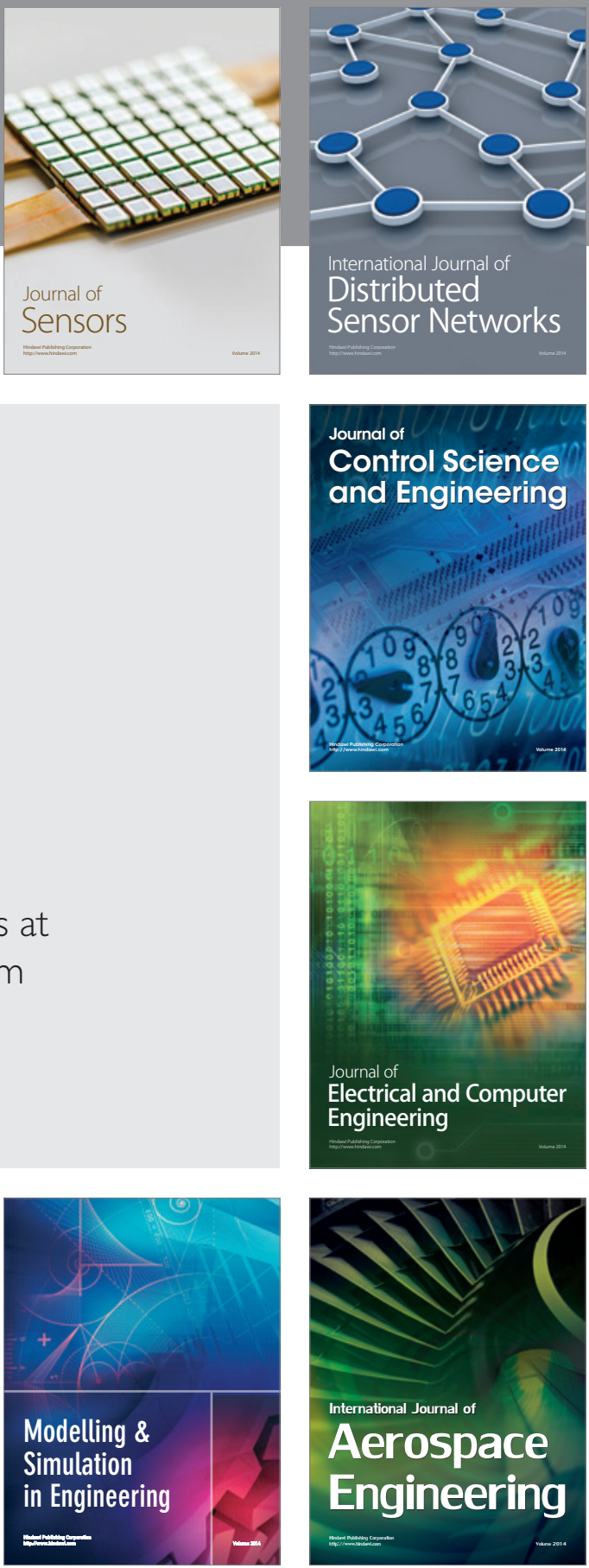

Journal of

Control Science

and Engineering
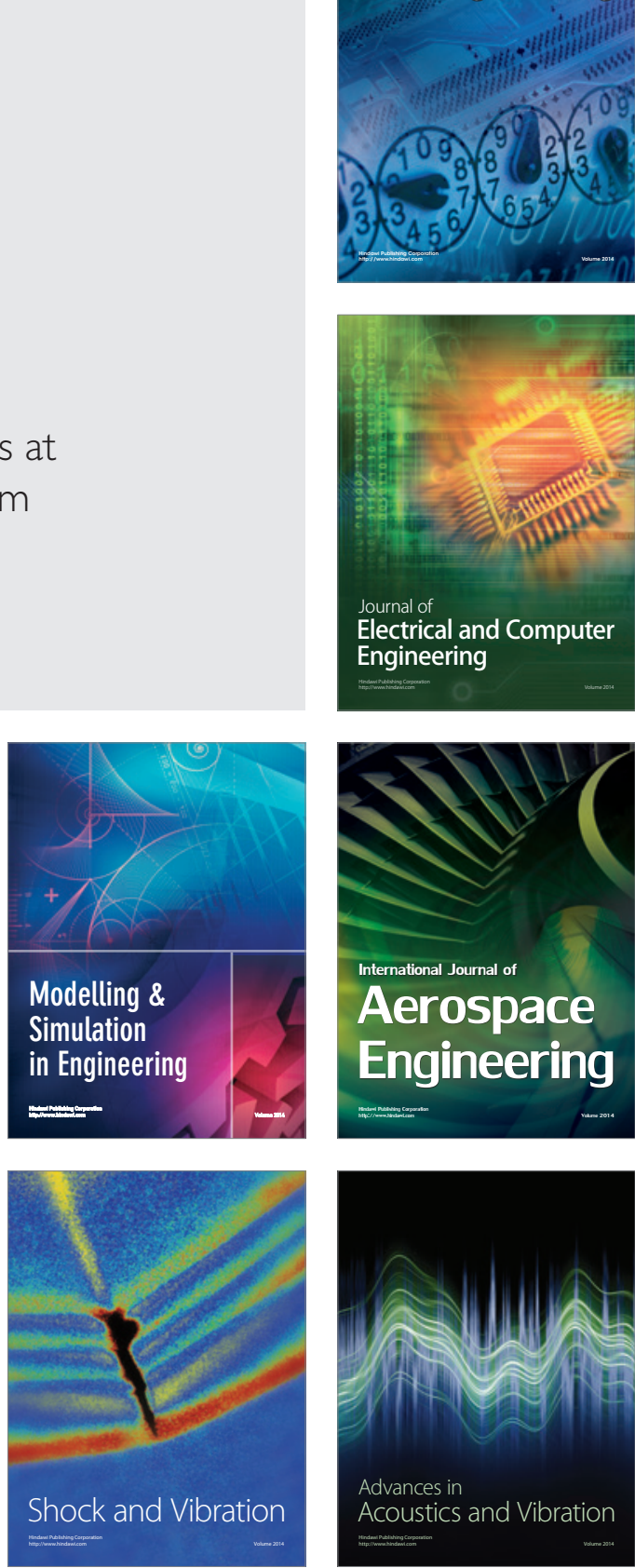